\title{
TAXONOMÍA y DISTRIBUCIÓN DEL GÉNERO SPARTINA (Poaceae) en México
}

\author{
Gabriela del Rocío Nieto-Silva ${ }^{1}$, María Elena Siqueiros-Delgado ${ }^{1,3}$, \\ José de Jesús Luna-RUiZ², ERnesto Flores-AnCiRA² y OnÉsimo Moreno-Rico ${ }^{1}$ \\ ${ }^{1}$ Centro de Ciencias Básicas, \\ ${ }^{2}$ Centro de Ciencias Agropecuarias, Universidad Autónoma de Aguascalientes, Aguascalientes, Aguascalientes, México \\ ${ }^{3}$ Autor para la correspondencia: masiquei@correo.uaa.mx
}

Resumen: Spartina es una gramínea peculiar por sus procesos de dispersión, sobrevivencia y evolución. Se desarrolla en ecosistemas de marismas, sobre suelos salinos en costas y en cuencas interiores. El género se encuentra distribuido principalmente en toda la costa Atlántica, en la costa Pacífica, únicamente en Baja California, Sinaloa y Oaxaca y en zonas interiores de Coahuila y San Luis Potosí. Todas las especies son poliploides, con una gran capacidad de hibridación y son usadas en la restauración de áreas con pérdida de suelo, convirtiéndose en muchos casos, en especies invasoras. Hasta la fecha, en México no se ha llevado a cabo un estudio exclusivo y exhaustivo sobre este género, por ello el objetivo del presente proyecto fue determinar el número de especies y distribución de Spartina en el país. En base a la revisión de ejemplares de herbario y bibliografía, se llevó a cabo la exploración de campo en las localidades donde se ha reportado la presencia de Spartina. En este estudio se reportan cuatro especies de las seis citadas para México: S. alterniflora, S. foliosa, S. patens y S. spartinae, siendo esta última la más ampliamente distribuida. Se ha señalado la presencia de $S$. gracilis en Jalisco, Michoacán y Chihuahua; y $S$. cynosouroides en Chihuahua y Tamaulipas, sin embargo, éstas no fueron localizadas en la exploración de campo ni en la revisión de herbario. No se encontró evidencia de especies introducidas en el país.

Palabras clave: Chloridoide, Poaceae, Spartina alterniflora, Spartina spartinae, suelos salinos.

\begin{abstract}
Spartina is a distinctive grass genus because of its processes of dispersal, survival and evolution. It grows in ecosystems of tidal marshes, mudflats and saline basins. The genus is distributed mainly along the entire Atlantic coast, on the Pacific coast only in Baja California, Sinaloa and Oaxaca, and in inland areas of Coahuila and San Luis Potosi. All species are polyploid with a great capacity for hybridization. Frequently species of Spartina have been used in restoration of areas with soil loss; however in many cases they have become invasive weeds. To date, there has not been carried out an exclusive and comprehensive study of this genus in Mexico. The aim of this project was to determine the number of species of Spartina and their distribution in the country. Based on bibliographic and herbarium voucher information, field explorations were carried out in all Mexican localities where Spartina had been reported. Four out of the six species reported for Mexico were found: S. alterniflora, S. foliosa, S. patens, and $S$. spartinae, the latter being the most widely distributed. Reports of $S$. gracilis in Jalisco and $S$. cynosuroides in Chihuahua were noted; however, these species were not located in field explorations nor in herbarium collections. There is no evidence of any introduced species.
\end{abstract}

Key words: Chloridoide, Poaceae, saline soils, Spartina alterniflora, Spartina spartinae.

$\mathbf{E}$ 1 género Spartina Schreb. es miembro de la familia Poaceae, subfamilia Chloridoideae y tribu Zoyseae (Peterson et al., 2001). Se desarrolla principalmente en ecosistemas de marismas sobre suelos salinos, tiene un hábito semi-acuático con un sistema de rizomas fuertes y profundos que lo hace un eficiente colonizador de áreas nuevas. Posee un sistema fotosintético $\mathrm{C} 4$, con tolerancia a altas temperaturas y salinidad, todas sus especies son poliploides y presentan una gran capacidad de hibridación (Ayres et al., 2008; Fortune et al., 2008). El género Spartina en su gran mayoría, es nativo del Continente Americano, sólo Spartina maritima Fernald es nativa de Europa y de las costas Atlánticas de África (Ainouche et al., 2009). De acuerdo a Mobberley (1956) existen 16 especies (considerando dos 
especies híbridas), sin embargo, de acuerdo a Ainouche et al., (2009) y Barkworth (2003), el número de especies varía de 13 a 15 y de 15 a 17, respectivamente. En México, se han reportado seis especies: Spartina alterniflora Loisel., distribuida en Chihuahua, Coahuila, Tamaulipas y Veracruz (Espejo-Serna et al., 2000; Mora-Olivo y Valdés-Reyna, 2011); Spartina cynosuroides (L.) Roth mencionada para Chihuahua y Tamaulipas (Espejo-Serna et al., 2000; Dávila et al., 2006; Herrera y Cortés, 2010); Spartina foliosa Trin. restringida a Baja California y Baja California Sur (Espejo-Serna et al., 2000; Dávila-Aranda et al., 2006); Spartina gracilis Trin. reportada para Chihuahua, Jalisco y Michoacán (Espejo-Serna et al., 2000; Dávila-Aranda et al., 2006; Herrera y Cortés, 2010); Spartina patens (Aiton) Muhl. localizada en Quintana Roo, Tabasco, y Tamaulipas (Espejo-Serna et al., 2000; Dávila-Aranda et al., 2006) y Spartina spartinae (Trin.) Merr ex. Hitchc. distribuida en toda la costa Atlántica desde Tamaulipas hasta Quintana Roo (Espejo-Serna et al., 2000; Dávila-Aranda et al., 2006), en Oaxaca, así como en cuencas endorreicas de Coahuila, Nuevo León y San Luis Potosí (Espejo-Serna et al., 2000; Peterson et al., 2001; Dávila-Aranda et al., 2006). Las especies de Sparti$n a$ tienen la particularidad de estructurar ambientes y crear refugios de estrés ambiental. A muchas de estas especies se les ha considerado como ingenieros ecológicos, debido a su rápida dispersión por medio de semillas y rizomas, formando densos pastizales donde los culmos y el tapetillo de raíces y rizomas son efectivos para filtrar y capturar sedimentos (Gedan y Bertness, 2010). Por lo anterior, algunas especies han sido introducidas para el control de erosión, sin embargo, existe el riesgo de romper el equilibrio de los estuarios por problemas de infestación con estas especies. Por ejemplo en Nueva Inglaterra, Estados Unidos, a través del dosel de sombra que produce $S$. patens y por la proliferación de sus raíces, se ha logrado reducir la salinidad, anoxia, desecación y estrés calórico del estuario (Gedan y Bertness, 2010). Otro ejemplo es S. alterniflora, especie nativa de Norte América y ampliamente utilizada en restauración de áreas con importante pérdida de suelo. Ésta especie ha producido organismos híbridos y se ha convertido en un invasor, lo cual representa un problema grave al desplazar a las especies nativas (Espinar, 2009). El género Spartina es un poliploide estricto, hasta el momento no se han localizado poblaciones diploides. Su número cromosómico es $\mathrm{x}=$ 10 , su nivel de poliploidía va desde tetraploides $(2 \mathrm{n}=4 \mathrm{x}=$ 40 en Spartina arundinaceae (Thouars) Carmich., Spartina bakeri Merr., S. cynosuroides, S. gracilis, S. patens, Spartina pectinata Link, $S$. spartinae), hexaploide $(2 \mathrm{n}=6 \mathrm{x}=$ 60 o 62 en S. alterniflora, Spartina densiflora Brongn., S. foliosa, Spartina glabra Muhl. ex Elliott, S. maritima) hasta dodecaploide $(2 \mathrm{n}=12 \mathrm{x}=122,124$ en Spartina ánglica C.E. Hubb.; Barkworth, 2003). Estos niveles de poliploidía se observan tanto entre especies, como al interior de la misma especie, por ejemplo $S$. pectinata la cual muestra 3 niveles de poliploidía en áreas donde se traslapan o intercalan sus poblaciones (tetraploides [ $2 \mathrm{n}=4 \mathrm{x}=40$ ], hexaploides [ $2 \mathrm{n}$ $=6 \mathrm{x}=60]$ y octoploides [2n $=8 \mathrm{x}=80$ ]; Kim et al., 2012). Spartina ha sido ampliamente estudiada desde el punto de vista ecológico y evolutivo, pero a nivel taxonómico tradicional su conocimiento está fundamentado en la revisión taxonómica de Mobberley (1956), basada en morfología. De acuerdo con este autor, a nivel mundial existen 16 especies de Spartina agrupadas en tres principales complejos de acuerdo a su morfología, y considerando como caracteres diagnósticos, la consistencia del culmo, el arreglo de las espiguillas, la forma de las raíces y su hábitat.

Estudios moleculares han confirmado la monofilia del género Spartina y han ayudado a dilucidar las relaciones filogenéticas entre sus especies (Baumel et al., 2002). Evidencias moleculares de nueve especies del género, confirman la presencia de dos principales linajes, uno formado por especies hexaploides y otro por especies tetraploides, revelando una estrecha relación entre S. alterniflora y $S$. foliosa (Baumel et al., 2002; Ainouche et al., 2003; Fortune et al., 2008). Así mismo, pone de manifiesto la concordancia de la hipótesis molecular de Baumel et al. (2002) con la clasificación tradicional de Mobberley (1956). Hipótesis filogenéticas de múltiples genes (Peterson et al., 2010) demuestran que la diversificación de Spartina se dio dentro de la misma línea evolutiva del género Sporobolus R. Br., por lo que con el fin de resolver Sporobolus como monofilético, las especies de Spartina fueron propuestas para ser tratadas como Sporobolus (Peterson et al. 2014)

En México, la información acerca del género Spartina es producto de colectas aisladas y fortuitas de diferentes investigaciones (Sosa y Gómez-Pompa, 1994) y de algunas floras regionales (Swallen, 1955; Kearney y Peebles, 1960; Shreve y Wiggins, 1964; Correll y Johnston, 1970; Correll y Correll, 1972; Gould y Moran, 1981; Espejo-Serna et al., 2000; Soreng et al., 2003; Barkworth, 2003). Sin embargo, hasta la fecha no se ha llevado a cabo un estudio ex profeso para conocer las especies, su distribución y su hábitat. Por lo tanto, el objetivo de este estudio fue llevar a cabo la revisión taxonómica de las especies de Spartina presentes en México, su distribución y determinar si hay especies introducidas en el país.

\section{Materiales y métodos}

Trabajo de campo. Los sitios de colecta se eligieron de acuerdo a las localidades referenciadas en las bases de datos del SNIB (Sistema Nacional de Información sobre la Biodiversidad), de la Comisión Nacional para el Conocimiento y Uso de la Biodiversidad (CONABIO), de la base de datos de la Red Mundial de Información sobre Biodiversidad (REMIB) de la CONABIO, y de las bases de datos de algunos de los herbarios consultados (CICY, INEGI, LL, TEX, UAT, $\mathrm{XAL}$ ). Derivado de esta información se tuvieron las siguien- 
tes zonas de colecta (Apéndice 1): Costa Atlántica (desde Tamaulipas a Quintana Roo), Costa Pacífica (Sinaloa, Baja California Sur y Chiapas) y la zona interior del país (Chihuahua, Coahuila y San Luis Potosí). Se colectaron al menos cinco ejemplares completos por sitio, incluyendo rizomas o raíces, hojas, culmos e inflorescencias, con los cuales se llevó a cabo la identificación taxonómica de la especie. Los ejemplares de herbario están depositados en el Herbario de la Universidad Autónoma de Aguascalientes (HUAA) y los duplicados se depositarán en el herbario del INEGI. Los datos de suelo, clima y vegetación del sitio se obtuvieron de la información geográfica del INEGI (2013). En cada sitio, en un formato de campo especial para el proyecto, se registraron datos de localidad, de tipo de vegetación, coordenadas y altitud con un GPS $e$ Trex ${ }^{\circledast}$ de Garmin $^{\circledast}$. Se tomaron fotografías de los ejemplares colectados, considerando el hábito de la planta, los detalles de la espiguilla, las hojas y los rizomas o la raíz, además del hábitat.

Trabajo de laboratorio. Se llevó a cabo en el HUAA, los ejemplares colectados se procesaron de acuerdo a Lot y Chiang (1986). La identificación del material se realizó utilizando los trabajos taxonómicos de Hitchcock (1971), Swallen y McClure (1955), Mobberley (1956), Shreve y Wiggins (1964), Correll y Johnston (1970), Correll y Correll (1972), Gould (1975), Wiggins (1980), Gould y Moran (1981), Pohl (1994) y Barkworth (2003). Así mismo, se revisaron ejemplares de 22 herbarios nacionales: ANSM, CHAP, CHIP,
CICY, EBUM, ENCB, FCME, HCIB, HUAA, IEB, IBUG, INEGI, MEXU, OAX, SERO, SLPM, UAMIZ, UAS, UAT, UCAM, UNL y XAL y seis extranjeros: UTC, ISC, LL, RSA, TEX y US (acrónimos del Index Herbariorum [IH]). También se consultó con especialistas de los diferentes estados quienes reportan localidades con Spartina. En algunos herbarios no se encontraron ejemplares de Spartina colectados en México (CHIP, IBUG, OAX, SERO), sin embargo se mencionan con el fin de enfatizar las pocas colectas del género en México.

La descripción de los taxa y la elaboración de las claves dicotómicas, se llevó a cabo mediante la observación y medición de estructuras morfológicas diagnósticas como la inflorescencia, las espiguillas, las láminas, los culmos y las lígulas, así como el hábitat. En las descripciones, además se incluyó la lista de ejemplares revisados y características del hábitat, así como fotografías de cada especie con un detalle de la espiguilla. Con los datos obtenidos de las coordenadas de los sitos de colecta, tanto de los ejemplares colectados como de los revisados en los herbarios, se elaboraron mapas digitales de distribución utilizando el software Arcmap de ArcGis v.10.

\section{Resultados}

Durante el desarrollo del proyecto se revisaron 190 ejemplares de herbario y se colectaron 171 ejemplares en campo. De las seis especies reportadas para México sólo se encon-

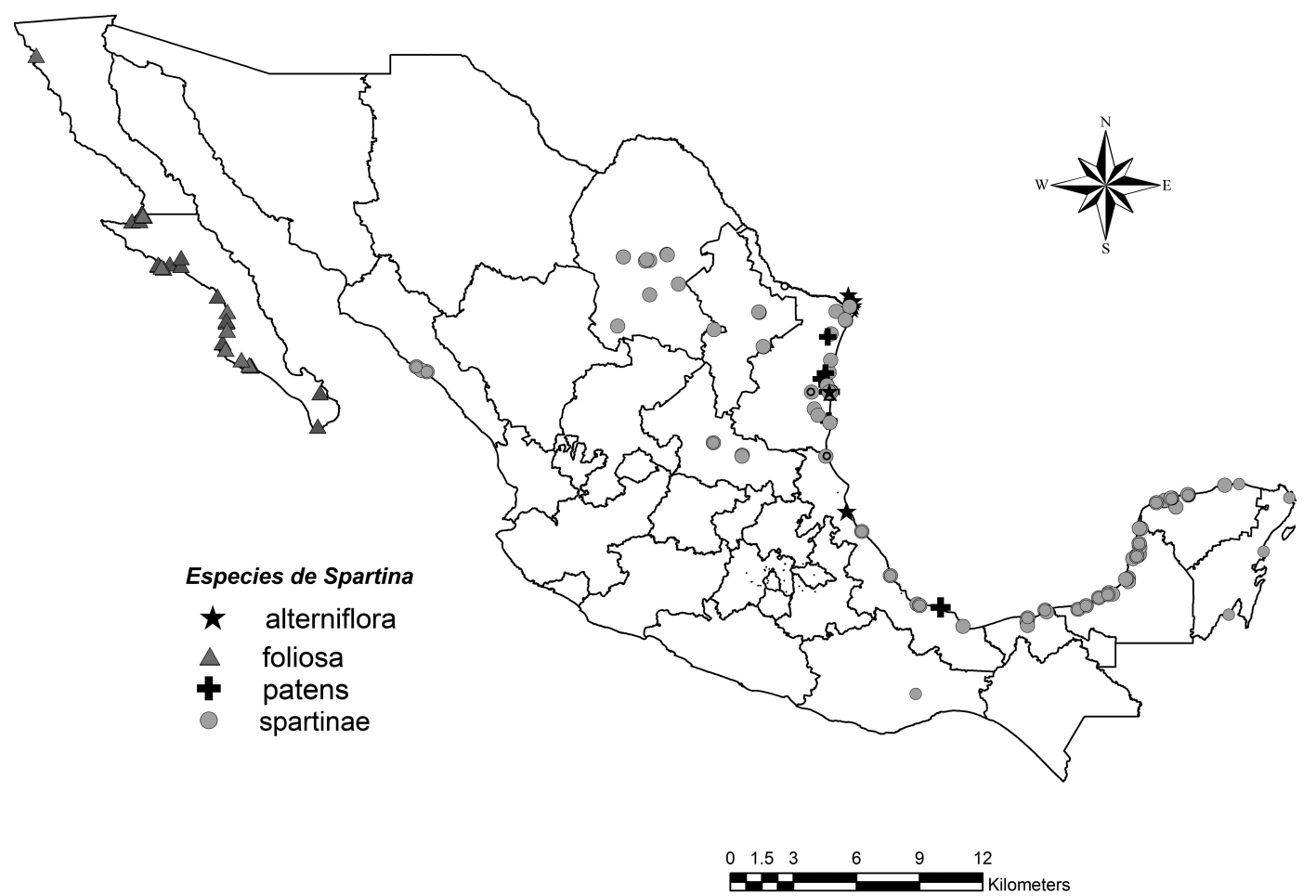

Figura 1. Distribución de la especies de Spartina en México de acuerdo a las colectas realizadas para este estudio y a la información obtenida en las revisiones de herbarios. 
traron cuatro: Spartina alterniflora, S. foliosa, S. patens y $S$. spartinae. No se encontraron registros de $S$. cynosuroides y $S$. gracilis en ningún herbario revisado ni en los recorridos de campo. La distribución de las especies de Spartina presentes en México se muestra en la Figura 1.

\section{Tratamiento taxonómico.}

Spartina Schreb. Gen. Pl. ed. 8[a]. 43 (1789). TIPO: Spartina cynosuroides (L.) Roth. Trachynotia Michx. Fl. Bor. Amer. 1:63. 1803. Limnetis Rich. in Pers. Syn. Pl.1:72. 1805. Ponceletia Thouars, Esquisse Fl. Tristan D’ Acugna. 36. 1808. TIPO: Ponceletia arundinacea Thouars. Tristania Poir. Encycl. 4: 526. 1816. Psammophila Schult. Mant.1:69, -231. 1822. TIPO: Psammophila arundinacea (Thouars) Schult. Mant. 1: 69, 231. 1822. Solenachne Steud. Syn. Pl. Glumac 1: 12. 1855 [1853]. TIPO: Solenachne phalaroides Steud. Chauvinia Steud. Syn. Pl. Glum. 1:362. 1854. TIPO: Chauvinia chilensis Steud.

Descripción.- Plantas perennes, la mayoría con rizomas, de hábitat acuático o semiacuático. Culmos erectos, glabros, carnosos o duros, solitarios a amacollados en grupos densos o laxos. Vainas glabras a pilosas; lígulas una hilera de tricomas; láminas planas o conduplicadas. Inflorescencia una panícula adpresa a abierta, terminal; raquis de la inflorescencia aplanado con varias espigas insertas subdigitalmente o a lo largo de éste, terminando en una prolongación estéril delgada, de 1.5-3.5 cm de largo, raquis de la espiga aplanado, ondulado, con una terminación estéril de 0.5-1.2. Espiguillas con un flósculo sésil, imbricadas, comprimidas lateramente, desarticulación por debajo de las glumas, arregladas en 2 hileras, raquis de las espigas glabros; gluma inferior más corta que el flósculo, 1-nervada, con quilla glabra, pilosa o híspida; gluma superior generalmente más grande que el flósculo, 1-3-nervada, quilla híspida, pectinada a glabra; lema de 1-3-nervada, quilla híspida a glabra; pálea de tamaño igual o ligeramente más larga que la lema 2-nervada, membranosa, quilla glabra; lodículas 2 o ausentes; estambres 3; estigmas 2. Cariopsis con el ápice terminando en una prolongación aguda.

Es un género nativo de América con 15-17 especies (incluyendo especies, subespecies e híbridos) la mayoría con distribución natural en Norte América (México y Estados Unidos de Norte América), una especie nativa de América del Sur (Spartina densiflora) y una especie nativa de Europa y África, S. maritima, la cual ha hibridado con especies introducidas originando un híbrido estéril (S. townsendii) y una nueva especie alopoliploide ( $S$. anglica) (Mobberley, 1956; Barkworth, 2003; Ainouche et al., 2009). Para México en este estudio se reportan cuatro especies.

Claves para identificación de las especies de Spartina reportadas para México en este trabajo
1 Culmos solitarios, carnosos, láminas planas, de 5-25 mm de ancho, generalmente sumergido en los márgenes de ríos o en zonas de mareas

2 Ápice de la lema usualmente acuminado, ramas de la inflorescencia de 7-20 cm de largo, glumas rectas, plantas nativas del Golfo de México (Tamaulipas y Veracruz)

........................................ alterniflora 2 Ápice de la lema redondo a obtuso, ramas de inflorescencia de $3.5-8 \mathrm{~cm}$ de largo, glumas curvas, plantas nativas del Pacífico Norte, California y Baja California S. foliosa

1 Culmos amacollados densa o laxamente, duros, no carnosos, láminas conduplicadas, de 1.5-6 mm de ancho, generalmente sobre arena o en zonas inundables, pero no sumergidos.

3 Ramas de la inflorescencia de 15-75, de 1-6.2 cm de largo, generalmente adpresas al raquis principal, culmos cespitosos formando grandes macollos, sin rizoma S. spartinae

3 Ramas de la inflorescencia 2-7, de 3-8 cm de largo, generalmente divergentes, culmos formando macollos delgados y laxos, ocasionalmente solitarios, con rizomas largos y delgados S. patens Spartina alterniflora Loisel. Fl. Gall. ii- 719. 1807.

=Trachynotia alterniflora DC., =Spartina stricta var. alterniflora (Loisel.) A. Gray, Spartina glabra var. alterniflora (Loisel.) Merr., झSpartina maritima var. alterniflora (Loisel.) St.-Yves. TIPO: Francia, sobre el camino a Bayonne, en pradera a lo largo de la costa, sin colector (Holotipo: AV). =Dactylis fasciculata Lam. TIPO: Estados Unidos de Norte América, D. Richard s.n. (Holotipo: P; Isotipo: US, fragmento en P).

$=$ Spartina brasiliensis Raddi, $\equiv$ Spartina maritima var. bra siliensis (Raddi) St.-Yves. TIPO: Brasil, Rio de Janeiro, G. Raddi s.n. (Holotipo: PI; Isotipo: BAA; Isolectotipo: FI; Isotipo: US).

$=$ Spartina glabra Muhl., =Spartina stricta var. glabra (Muhl . ex Elliott) A. Gray, Limnetis glabra (Muhl. ex Elliott) Eaton \& Wright, 三Spartina alterniflora var. glabra (Muhl. ex Elliott) Fernald, Spartina maritima subsp. glabra St.-Yves, $\equiv$ Spartina arítima var. glabra St.-Yves. TIPO: Estados Unidos de Norte América, Carolina del Sur o Georgia, S. Elliott s.n. (Holotipo: CHM).

=Spartina glabra var. pilosa Merr., 三Spartina maritima subvar. pilosa (Merr.) St.-Yves. TIPO: Estados Unidos de Norte América, New Jersey, F.L. Scribner s.n. (Isotipo: US).

$=$ Spartina laevigata Bosc ex Link. TIPO: Estados Unidos de Norte America, Link s.n. (Isotipo: US).

=Spartina merrillii A. Chev. TIPO: Estados Unidos de Norte América, New York: Long Island, Rockaway Point, E. P. Bicknell 11300 (Holotipo: NY; Isotipo: PH).

Descripción.- Plantas acuáticas, sumergidas en los márgenes de ríos o en zonas de mareas, con rizomas largos, las 
escamas algo infladas o expandidas y no muy imbricadas. Culmos carnosos, principalmente solitarios, o en grupos pequeños, erectos de $70-270 \mathrm{~cm}$. Vainas glabras con la garganta glabra a laxamente pilosa; lígulas de $3 \mathrm{~mm}$ de largo; láminas planas, glabras, de $30-52 \mathrm{~cm}$ de largo, $10-25 \mathrm{~mm}$ de ancho, haz acostillado, envés glabro, ápice acuminado, terminando en una punta aguda. Inflorescencia una panícula adpresa, $10-40 \mathrm{~cm}$ de largo y hasta $2 \mathrm{~cm}$ de ancho, con 2-20 ramas de 7-20 cm de largo; espiguillas de 10-30 por rama, adpresas, de 10-14 mm de largo con ápice agudo, raquis de las espigas frecuentemente prolongado más allá de la espiguilla terminal; gluma inferior 1-nervada, de 4-6 $\mathrm{mm}$ de largo, aguda, usualmente recta, glabra a esparcidamente pilosa, quilla pilosa; gluma superior 3-nervada, de 8-14 mm de largo, acuminada a obtusa, usualmente recta, quilla glabra a pilosa; lema 3-nervada, 10-14 mm de largo, glabra a esparcidamente pilosa, ápice usualmente acuminado, quilla glabra; pálea 2-nervada, de 9-11 mm de largo, delgada, membranosa, obtusa a redondeada. Cariopsis no vista (Figura 2A).

Morfológicamente, Spartina alterniflora es parecida a $S$. foliosa. Mobberley (1956), incluye estas dos especies en el mismo complejo y datos moleculares indican que son especies hermanas (Baumel et al., 2002). El carácter diagnóstico que las diferencia es el ápice de la lema acuminado en $S$. alterniflora y obtuso en S. foliosa, además de la distribución. Distribución y ecología.- De forma natural se distribuye en las costas del océano Atlántico desde Canadá hasta Argentina (Mobberley, 1956; Barkworth, 2003; Ainouche et al., 2009; Saarela, 2012). En México se ha encontrado en Tamaulipas y Veracruz. Suele estar sumergida en aguas salobres en los márgenes de estuarios y ríos o en zonas de mareas (Mora-Olivo y Valdés-Reyna, 2011). Esta especie generalmente forma densas poblaciones monoespecíficas, o en asociación con Rhizophora mangle L., Laguncularia racemosa (L.) C.F. Gaertn. y Avicennia germinans (L.) L., en donde es importante como refugio y alimento para la fauna silvestre. Sin embargo, en lugares donde ha sido introducida como restauradora de zonas perturbadas en Asia (China), Europa, Australia y sur de los Estados Unidos de Norte América compite con las especies nativas (Baumel et al., 2003; Zhi et al., 2007; Ayres et al., 2008; Hogle, 2011).

Ejemplares examinados.- Tamaulipas: Mpio. Matamoros, Río Bravo, Los Chorreaderos, $1.5 \mathrm{~km}$ al SO de la desembocadura, Mora-Olivo 11142 (UAT); Mpio. Soto La Marina, $0.8 \mathrm{~km}$ al SW de Las Escolleras, Rojas 692 (INEGI); Área urbana ejido La Pesca, orilla del estero, Brito 162 (MEXU); Río Soto La Marina, Ejido La Pesca, Mora-Olivo 5429 (MEXU, UAT); Río Soto La Marina, La Pesca, Nieto 369 370, 371, 372, 373 (HUAA); Río Soto la Marina, Vista Hermosa, Iribe 514 (UAT). Veracruz: Mpio. Tuxpan, Laguna de Tampamachoco, cerca Río Tuxpan, Sandoval 403 (XAL); Vega del Río Pantepec, Brigada Veracruz 246
(MEXU); Vega del Rio Tuxpan, Malecón Norte en la ciudad de Tuxpan, Nieto S. 318, 319, 320, 321, 322 (HUAA).

Spartina foliosa Trin. Mém. Acad. Imp. Sci. Saint-Pétersbourg, Sér. 6, Sci Math., Seconde Pt. Sci. Nat. 6,4(1-2): 114. 1840.

=Spartina stricta Roth var. foliosa (Trin). Thurb. TIPO: Estados Unidos de Norteamérica, California, D. Peters s.n. (Isotipo: US, fragmento en LE).

=Spartina densiflora fo. acuta St.-Yves. TIPO: Estados Unidos de Norte América, California: Condado de Humbodlt, A. A. Heller 13781 (Isotipo: US).

=Spartina leiantha Benth. TIPO: México, Baja California Sur: Bahía de Magdalena, sin colector (Isotipo: US, fragmento en BM).

Descripción.- Plantas acuáticas, sumergidas en los márgenes de ríos o en zonas de mareas, con rizomas largos y las escamas algo infladas, no muy imbricadas. Culmos hasta de $1.5 \mathrm{~m}$ de alto, erectos cilíndricos, carnosos, glabros, frecuentemente con raíces adventicias en los nudos inferiores del culmo, usualmente solitarios o en grupos pequeños. Vainas glabras; lígulas de $2 \mathrm{~mm}$ de largo; láminas planas a ligeramente involutas, glabras, $16-40 \mathrm{~cm}$ de largo y 5-12 $\mathrm{mm}$ de ancho. Inflorescencia una panícula adpresa, de 14$25 \mathrm{~cm}$ de largo por $1 \mathrm{~cm}$ de diámetro, con 6-14 ramas de $3.5-8 \mathrm{~cm}$ de largo; espiguillas $10-35$, de $9-12 \mathrm{~mm}$ de largo; gluma inferior 1-nervada, de 6-9 mm de largo, acuminada a obtusa, usualmente curva, glabra a pilosa en la quilla; gluma superior 3-nervada, nervaduras apenas perceptibles, de 10-17 mm de largo, ápice agudo a obtuso, terminado en un mucrón diminuto o prolongación aguda de la vena media, usualmente curva, quilla glabra; lema 3-nervada, de 8-10 $\mathrm{mm}$ de largo, ápice redondo a obtuso, quilla glabra; pálea 2-nervada, de 8-12 mm de largo, glabra. Cariopsis no vista (Figura 2B).

Distribución y ecología.- Spartina foliosa se concentra en los manglares o zonas intertidales desde la Bahía de California a las costas de la Península de Baja California por el Océano Atlántico (Mobberley, 1956; Barkworth, 2003), codominando con Conocarpus erectus L. y asociado a Batis maritima L. y Allenrolfea occidentalis (S.Watson) Kuntze, o bien formar grandes pastizales halófilos. Aunque está ampliamente distribuida en la Península de Baja California, en algunos sitios su hábitat natural ha sido destruido para el establecimiento de zonas turísticas, especialmente en la región de los Cabos. No se encontró en la costa del Mar de Cortés de la Península de Baja California a pesar de la exploración de campo.

Ejemplares examinados.- Baja California: Mpio. Ensenada, $10 \mathrm{~km}$ al NW de Guerrero Negro, Carr. a Puerto Viejo, Díaz R. 21 (INEGI, XAL); Bahía de San Quintín, Dawson 21582 (US); Boca de Estero Beach, en los límites 


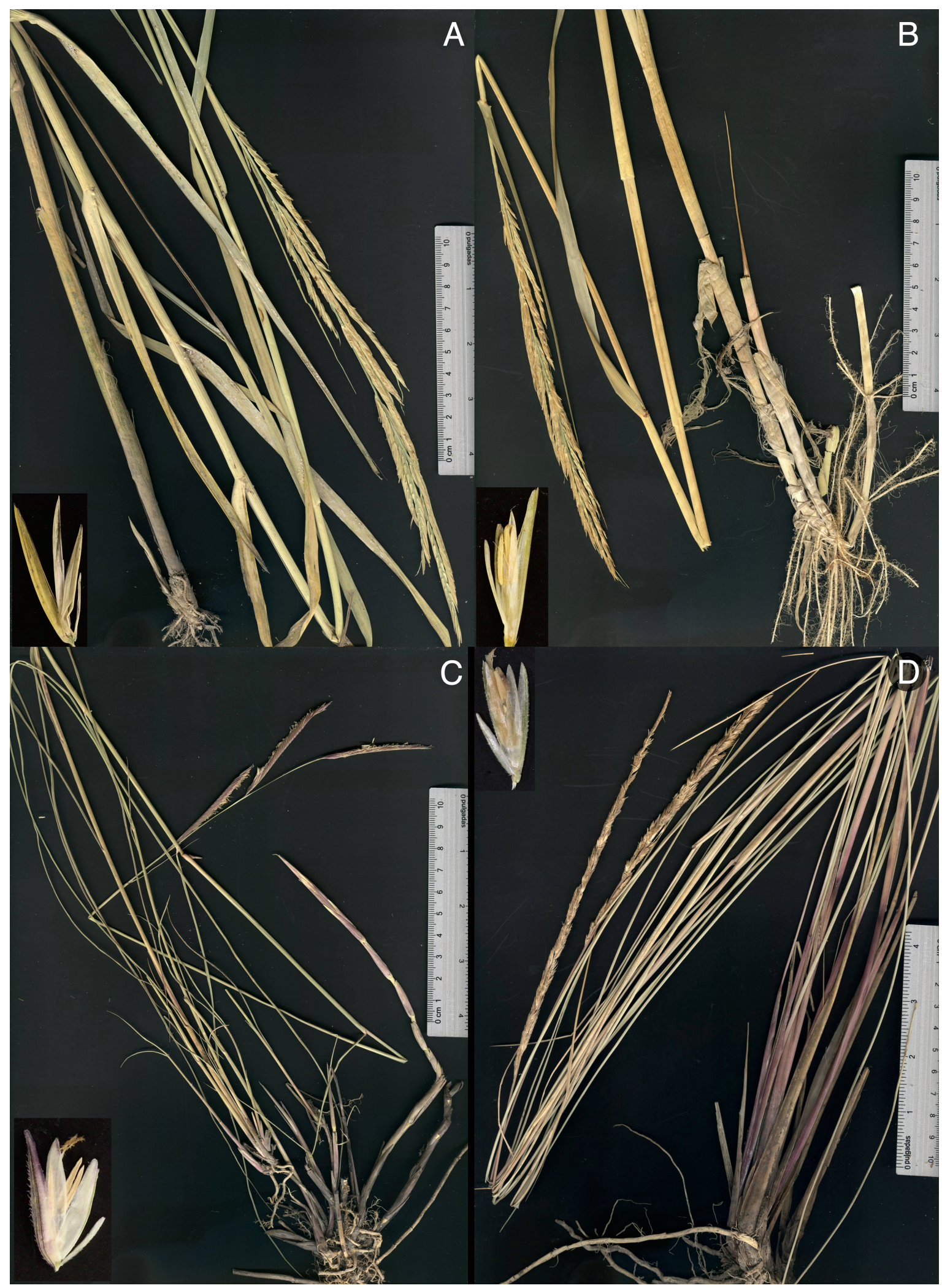

Figura 2. Fotografías de las cuatro especies de Spartina distribuidas en México. A) Spartina alterniflora con detalle de la espiguilla, La Pesca, Tamps., G. Nieto 369. B) Spartina foliosa con detalle de la espiguilla, Puerto Chale, BCS, G. Nieto 169. C) Spartina patens con detalle de la espiguilla, Río Soto la Marina, Tamps., G. Nieto 364. D) Spartina spartinae con detalle de la espiguilla, Puerto Progreso, Yuc., G. Nieto 216. 
sur de la Ciudad de Ensenada (enfrente del Hotel-Resorts Estero Beach y la playa de Estero Beach), Rodríguez s.n. (HUAA); Campamento Las Grullas, Estero de Punta Banda cerca de Ensenada, Guzmán del Próo s.n. (ENCB, POLI); Punta Banda, Ibarra L. 1 (INEGI). Baja California Sur: Costa del lago Scammon, Reeder \& Reeder 6652 (ENCB, US, RSA, TEX); Mpio. Comondú, Bahía Magdalena Estero de San Jorge, León 552 (HCIB); Bahía de Magdalena, Isla Magdalena, $5.2 \mathrm{~km}$ al S de Boca de Santo Domingo, Medel et al. 11-189 (HCIB); Bahía de Magdalena, Islote de arena, $1 \mathrm{~km}$ al N de boca de Santo Domingo, Pérez 567 (HCIB); Isla Magdalena, hacia la parte media de la isla, Pérez 621 (HCIB); Lado E de Bahía Magdalena 0.7 mi al NE de San Carlos, Burgess et al. 6161 (ENCB); Playa San Carlos, Reeder y Reeder 6623 (ENCB, POLI, TEX); Puerto Adolfo López Mateos, estero en el margen del manglar, Nieto S. 183, 184, 185, 186, 187, 188 (HUAA); Puerto San Carlos, entrada E del Pueblo, carr. Constitución-San Carlos, playa a la entrada, Nieto S. 176, 177, 178, 179, 180, 181, 182 (HUAA); Punta Isla Magdalena, Ramos \& López F. 612 (TEX). Mpio. La Paz, El Dátil, manglar del campamento pesquero el Dátil, Pérez 473 (HCIB); Isla Mangle, frente a la Isla Margarita, Bahía Magdalena, Holguin et al., s.n. (ENCB, POLI); Puerto Cancún $0.5 \mathrm{~km}$ al $\mathrm{W}$ del Pueblo, $32 \mathrm{Km}$ del entronque las Brisas Carr. La Paz-Cd. Constitución, Nieto S. 171, 172, 173, 174, 175 (HUAA); Puerto Chale, Estero de Puerto Chale, frente a la orilla del Estero, Nieto S. 164, 165, 166, 167, 168, 169, 170 (HUAA); Mpio. Mulegé, 3 km NW Guerrero Negro. carr. a Puerto Viejo, Malaquías 34 (INEGI); $6.8 \mathrm{~km}$ al NW de Punta Abreojos, estero La Bocana, extremo sur márgenes del estero, Nieto S. 189, 190, 191, 192, 193 (HUAA); Canal que cruza la $\mathrm{Cd}$. de Guerrero Negro, perpendicular a la calle principal sobre los márgenes del canal, Nieto S. 194, 195, 196, 197 (HUAA); Cerca del campamento de las salinas en Guerrero Negro, Thomas 8263 (ENCB); Guerrero Negro Desviación a la Playa, antes de la salinera, Vargas y Ortiz 100 (CHAPA, ENCB); Laguna Abreojos, 2 millas al SE de la Bocana, Moran 22760 (ENCB); Laguna Ojo de Liebre, $5 \mathrm{~km}$ al NW del pueblo de Guerrero Negro, Brecha al Faro Viejo, Nieto S. 198, 199, 200, 201, 202, 203 (HUAA); NW de Abreojos, Morán \& Reveal 19743 (US).

Spartina patens (Aiton) Muhl. Descr. Gram. 55, 1817. =Dactylis patens Aiton., झSpartina juncea var. patens (Aiton) St.-Yves. TIPO: Reino Unido, cultivada, Curtis s.n. (Holotipo: BM).

=Limnetis juncea var. monogyna M.A. Curtis, =Spartina patens var. monogyna (M. A. Curtis) Fernald. TIPO: Estados Unidos de Norte América, Carolina del Norte, Curtis s.n. (Isotipo: GH).

=Spartina pumila Roth, TIPO: Estados Unidos de Norte América, Nueva York, Schreber s.n. (Holotipo: B).

=Trachynotia juncea Michx., झLimnetis juncea (Michx.)

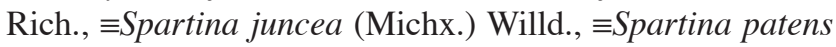

var. juncea (Michx.) Hitchc. TIPO: Estados Unidos de Norte América, Carolina meridional, orillas de estuario en la Base Carolina, Michaux s.n. (Holotipo: P).

Descripción.- Plantas semiacuáticas a terrestres, con rizomas largos y delgados pero fuertes, las escamas imbricadas, frecuentemente rojizas. Culmos de $10-90 \mathrm{~cm}$ de alto formando macollos delgados y laxos con pocos culmos duros, no carnosos, ocasionalmente solitarios. Vainas glabras; lígulas de $0.5 \mathrm{~mm}$ de largo; láminas conduplicadas algunas veces planas, de $40-50 \mathrm{~cm}$ de largo y $1.5-3 \mathrm{~mm}$ ancho, haz escabroso, envés glabro. Inflorescencia una panícula cilíndrica de $12-17 \mathrm{~cm}$ de largo y de $0.5-1 \mathrm{~cm}$ de diámetro, con 2-7 ramas por panícula, de 3-8 cm, alternas, difiriendo sólo ligeramente en longitud y espaciado dentro de la panícula, generalmente divergentes y en ocasiones adpresas; espiguillas 10-30 de 7-12 mm, linear lanceoladas a ovado lanceoladas; gluma inferior 2-nervada, 3-8 mm, linear, acuminada, quilla híspida; gluma superior con 2 venas laterales, 7-12 $\mathrm{mm}$, acuminada, quilla escabrosa a híspida; lema 3-nervada, de 5-7 mm, obtusa o redonda o inconspicuamente lobada, quilla híspida distalmente; palea 2-nervada, 5-6 mm, membranosa, ápice redondeado; indehiscente, glabra a esparcidamente híspida. Cariopsis no observada en los ejemplares examinados (Figura 2C).

Distribución y ecología.- Spartina patens se desarrolla en zonas costeras salinas de Norte América (Quebec a Florida y Golfo de México), extendiéndose a través de las Islas Caribeñas y en las costa norte de Sudamérica, se encuentra como introducida en España, Francia, Italia y en China (Mobberley, 1956; Gould, 1975; Barkworth, 2003). En México se encuentra distribuida en Tamaulipas y Veracruz, en zonas de manglar o pastizales halófilos, sobre arena o en zonas inundables, pero no sumergida, generalmente asociada a manglares de Laguncularia racemosa, en ocasiones cohabitando con S. spartinae. En la revisión de herbarios se pudieron detectar algunos errores en la identificación de este taxón, lo que puede conducir a confusión en la distribución de la especie. Los ejemplares Susana Torres 259 (FCME), colectado en Mezcala, Guerrero y Jorge Martínez G. 250 (FCME), colectado en Zumpango del Río, Guerrero, ambos identificados como $S$. patens, son en realidad Bouteloua dimorpha J.T.Columbus.

Ejemplares examinados.- Tamaulipas: Mpio. Aldama, Laguna de San Andrés, coastal dunes near Morón, LeSueur 671 (TEX, UAT); Mpio. Matamoros, Playa, $5 \mathrm{mi} \mathrm{S}$ of mouth of Rio Grande, Hildebrand 8 (TEX, UAT); Mpio. San Fernando, Laguna Madre, vicinity of Media Luna, fishing Village SE of Santa Teresa, Fryxell 3733 (UAT); Mpio. Soto la Marina, $4 \mathrm{~km}$ al E del Poblado La Pesca, Baro 307 (CICY, ENCB, ANSM, IEB); Brecha junto al campamento tortuguero, Playa Grande, La Pesca, Nieto S. 374, 375, 
376, 377, 378 (HUAA); La Pesca, Puig, 2908 (ENCB); La Playita, margen del estero, La Pesca, Nieto S. 363, 364, 365, 366, 367, 368 (HUAA); Playa La Pesca, Baro 2817 (UAT). Veracruz: Mpio. Mecayapan, Pilapillo, $2.5 \mathrm{~km} \mathrm{NE}$ de Pilapillo, playa Boca Guayabillo, Castillo-Campos et al. 12669 (IEB).

Spartina spartinae (Trin.) Merr. ex Hitchc. Contr. U.S. Natl. Herb 17(3): 329. 1913.

=Vilfa spartinae Trin. TIPO: Estados Unidos de Norte América, Texas, Hooker s.n. (Holotipo: LE).

=Spartina argentinensis Parodi., $\equiv$ Spartina densiflora subvar. argentinensis (Parodi) St.-Yves. TIPO: Argentina, Santa Fe: Las Colonias, G. Niedfeld s.n. (Isosintipo: BAA; Isotipo: SI).

=Spartina densiflora var. obtusa Hack. TIPO: Paraguay: Gran Chaco, Hassler 2621 (Isotipo: US, fragmento en WU). =Spartina gouinii E. Fourn. TIPO: México, Veracruz, Gouin 72 (Isotipo: US, fragmento en P).

$=$ Spartina junciformis Engelm. \& A. Gray. $\equiv$ Spartina densiflora var. junciformis (Engelm. \& A. Gray) St.-Yves. TIPO: Estados Unidos de Norte América, Texas, Lindheimer 207 (Isotipo: GH; Holotipo: BM; Isotipo: MO).

=Spartina pittieri Hack. Oesterr. Bot. Z. 52: 237. 1902. TIPO: Costa Rica, Puerto Limon, Pittier 4209 (Holotipo: BR).

Descripción.- Plantas semiacuáticas a terrestres, sin rizomas, con raíces gruesas. Culmos de 60- $237 \mathrm{~cm}$ de alto, cespitosos, duros, no carnosos, formando densos macollos hasta de más de $50 \mathrm{~cm}$ de diámetro, erectos, generalmente glabros. Vainas glabras; lígulas de 0.6-1.5 mm de largo; láminas conduplicadas de $33-140 \mathrm{~cm}$ de largo y de $2-6 \mathrm{~mm}$ de ancho, haz estriado y escabroso, envés liso y glabro, margen inconspicuamente escabroso, ápice con punta espinosa. Inflorescencia una panícula de $20-48 \mathrm{~cm}$ de largo y de $0.5-1 \mathrm{~cm}$ de ancho, con 15-75 ramas, adpresas al raquis y algo recurvadas, de 1-6.2 cm de largo; espiguillas de 11-40 por rama, de 5.5-7 mm de largo; gluma inferior 1-nervada, de 2-5 $\mathrm{mm}$ de largo, acuminada, quilla híspida, con tintes morados hacia el ápice; gluma superior 3-nervada inconspicuamente, de 5-7 mm de largo, glabras a hispidulosas, acuminadas a obtusas, quilla híspida a escabrosa; lema 3-nervada, de 4-8 mm de largo, algunas veces con un mucrón terminal de $0.3 \mathrm{~mm}$, quilla híspida; palea 2-nervada, de 4-9 mm de largo, glabra, anteras 3-5 mm de largo, amarillas. Cariopsis glabra, de 4 $\mathrm{mm}$ de largo, con el ápice agudo terminando en una estructura espinescente de $2 \mathrm{~mm}$ de largo (Figura 2D).

Es la especie más fácil de diferenciar principalmente por el tamaño de sus macollos y por las numerosas ramas adpresas de la inflorescencia, además de carecer de rizoma.

Distribución y ecología.- Se encuentra en una gran diversidad de tipos de vegetación principalmente en manglares, mezquitales y en pastizales halófilos; en suelos salinos, en zonas inundables y en zonas con poca humedad, nunca sumergidas. Suele estar en asociación con Borrichia frutescens (L.) DC., Rhizophora mangle, Laguncularia racemosa y Avicennia germinans. Spartina spartinae es nativa del continente Americano. En Norte América, se distribuye en la costa Atlántica de Florida, Texas y México, en Centro América está presente en Costa Rica, y en Sudamérica en Paraguay y norte de Argentina, tanto en zonas costeras como en áreas halófitas interiores (Mobberley, 1956; Pohl, 1994; Barkworth, 2003). En México es la especie más abundante, encontrándose en las zonas costeras del Golfo de México desde Tamaulipas hasta Quintana Roo; en el Pacífico fue colectada únicamente en Sinaloa y se revisaron ejemplares de herbario colectado en zonas interiores de Oaxaca. Se establece en manglares y pastizales halófilos en las costas, así como en zonas salinas interiores de Coahuila y San Luis Potosí.

Los nombres comunes de esta especie en las diferentes partes del país son: "esparto", "xacahuite" o "pasto salado" en Tamaulipas, Veracruz, Tabasco, Coahuila y San Luis Potosí; en Tabasco y Campeche también se le conoce como "pincha huevos". En la región la utilizan para techo de palapas y lo podan o lo queman para dar los rebrotes como alimento al ganado.

Ejemplares examinados.- Campeche: Mpio. Calkiní, $2 \mathrm{~km}$ después del Remate, Chan V. y Burgos C. 650 (CICY, XAL); $11 \mathrm{~km}$ al SE de la Reserva de la Biósfera Los Petenes (terracería a la Isla Jania) Nieto S. 343, 344, 345, 346, 347 (HUAA); Mpio. Campeche: $0.5 \mathrm{~km}$ al NE de Campeche (por la salida a Carr. Campeche-Mérida), Nieto S. 209, 210, 211, 212 (HUAA); $1 \mathrm{~km}$ al NE de Lerma, Gutiérrez B. 7164 (CICY, MEXU); $2 \mathrm{~km}$ al NE de la Ciudad de Campeche, Gutiérrez, 7537 (CICY, UCAM, XAL); $2.5 \mathrm{~km}$ al O de San Fco. Kobén, Gutiérrez B. 7912 (UCAM, XAL); IMI NE de la Cd. de Campeche, Gutiérrez B. 7014 (CICY, UCAM), 9326, 9460 (UCAM); Las Palmas, Gutiérrez B. 5912 (CICY, MEXU); Margen izquierdo, $\mathrm{km} \mathrm{3.5,} \mathrm{carretera} \mathrm{Campeche-}$ Hampolol, Martín 357 (MEXU); Mpio. Carmen, 5 km NE de Sabancuy, Gutiérrez B. 8111 (UCAM); $10 \mathrm{~km}$ al E de Cd. del Carmen (frente a la finca Mayaraki) km 19 de la carr. Cd. del Carmen-Campeche, Nieto S. 338, 339, 340, 341, 342 (HUAA); $13 \mathrm{~km}$ al E de Sabancuy, Abnal M. 85 (INEGI); Ejido Puerto Rico, $7 \mathrm{Km}$ al N del Zacatal, km 16 carr. Villahermosa-Cd. del Carmen, Nieto S. 228, 229, 230 (HUAA); Mpio. Champotón, $0.5 \mathrm{~km}$ al S de Sabancuy (orillas del pueblo de Sabancuy por la salida a Chicbul), Nieto S. 353, 354, 355, 356, 357 (HUAA); $2 \mathrm{~km}$ al E de la ciudad de Champotón, Gutiérrez B. 7965 (MEXU, XAL); Camino a El Zapotal, $4 \mathrm{~km}$ desde el entronque con la carretera Campeche-Champotón Puch T. 49 (CICY, MEXU, XAL); km 53 de la autopista Cd. del Carmen-Champoton, Nieto S. 223, 224, 225, 226, 227 (HUAA); Mpio. Tenabo, $21 \mathrm{~km}$ al oeste de Tenabo, Abnal M. 20 (INEGI); Durán G. 1339 (XAL). Coahuila: Mpio. Cuatrociénegas, Coronado S. s.n. (INE- 
GI); Huerta A. et al. 40 (INEGI); Marroquín, 2671 (ANSM); Marsh 2037, 2079 (TEX)); 5.9 (rd) mi SW de Cuatrociénegas, a lo largo de la carretera 30, Henrickson \& Sundberg 7955 (TEX); 7 km S de la cuenca Cuatrociénegas en el pozo Mesquite, Henrickson \& Prigge 15368 (ANSM, TEX); 8 km al E de Cuatro Ciénegas, Rzedowski 26772 (POLI); 31 mi al E de Cuatrociénegas, Gould 15587 (POLI); Balneario los Mezquites, Brigada III, 12 (EBUM, ENCB, IEB, INEGI, MEXU), Dunas cercanas a la poza de la Borrega, Villareal et al. 3201 (ANSM); Dunas de Cuatrociénegas de Carranza entrada por la Poza de la Becerra, Ortiz s.n., (ANSM); E de Laguna de El Mojarral, Letho et al. 5162 (ENCB); Laderas de Sierra San Marcos, 24 mi al SO de Cuatrociénegas, Peterson 10009 (ANSM); Posa Azul, Siqueiros 4801 (HUAA); Pozo de la Becerra, Santana F. s.n. (ANSM); W laguna de El Mojarral, Cole et al. 3585 (LL); Mpio. Nadadores, $6 \mathrm{~km}$ E de Celemania (entre Nadadores y Sacramento), Johnston et al. 11227 (MEXU, TEX); a lo largo del Rio Salado, 2 millas al S de Hermanas, Reeder y Reeder 4568 (ENCB, US). Nuevo León: Mpio. Linares, Baño de San Ignacio, $22 \mathrm{~km}$ al NE de Linares, Cabral 656 (ANSM); Mpio. China, $4 \mathrm{~km}$ al N del Rancho El Divisadero, Valdés R. 181 (ANSM, UNL). Oaxaca: Mpio. Juchitán de Zaragoza, 6 km adelante de la Ventosa, rumbo a Chiapas, Beetle 5103 (MEXU); $10 \mathrm{~km}$ al SE de la Ventosa, Téllez et al. 30188 (MEXU, SLPM); Mpio. Santo Domingo Zanatepe, canal de irrigación, $1.8 \mathrm{mi}$ de la carr. 190, oeste de la desviación a Santo Domingo, Trott y Cols. 227 (ENCB, MEXU); Sabana cerca ing. Sto. Domingo, Miranda 8560 (MEXU). Quintana Roo: Mpio. Felipe Carrillo Puerto, Cayo Cedros, S de Punta Allen, Bahía de la Asunción, Cabrera C. 3406 (ENCB, MEXU, POLI, XAL); Vigía Chico, Téllez y Cabrera 3167 (MEXU). San Luís Potosí: Pringle 3451 (US); Mpio. Ciudad del Maíz, $0.65 \mathrm{Km}(0.4 \mathrm{mi}) \mathrm{N}$ of the Escuela Primaria en Las Tablas, entre las carreteras 70 y 80, Lane y Fryxell 2538-A (TEX); $2 \mathrm{~km} \mathrm{~N}$ de Las Tablas, $9.5 \mathrm{~km} \mathrm{~S}$ de Moritas al SW de Ciudad del Maíz, Siqueiros 4902, 4903, 4904 (HUAA); $10.5 \mathrm{~km}$ al S, SW de La Morita, Loera S. et al. 38 (INEGI, XAL); Las Tablas, Rzedowski 5837 (SLPM, US); Mpio. Rio Verde, 4 km sobre la terracería San Bartolo-Las Tablas, García s.n. (SLPM); Hacienda de Angostura Pringle 3760 (ENCB, MEXU, US). Sinaloa: Mpio. Culiacán, 15 $\mathrm{km}$ al W del Dorado (sobre brecha a los Algodones), playa de Ponce, Nieto S. 159, 160, 161, 162, 163 (HUAA); El Conchal, $0.2 \mathrm{~km}$ al W de la carr. al Conchal, a la entrada $\mathrm{S}$ del pueblo, Nieto S. 204, 205, 206, 207 (HUAA); Península de Lucenilla a $1 \mathrm{~km}$ de la entrada de la península, Hernández et al. 241 (MEXU, UAS). Tabasco: Mpio. Centla, 7 km al $\mathrm{N}$ de Frontera (Carr. A Frontera - Playas el Bosque), Nieto S. 231, 232, 233 (HUAA); Barra de San Pedro, cerca límites Campeche-Tabasco, Hernández X y Segalen 248 (MEXU); Mpio. Paraíso, $1.5 \mathrm{~km}$ al S de Chiltepec en la Laguna Mecoacán (por la carr. Chiltepec-Nicolás Bravo), Nieto S. 234, 235, 236, 237 (HUAA). km 20.6 en la carr. de Nicolás Bra- vo hacia Mecoacán a 26.4 km del carr. Paraíso-Comalcalco, orilla de la Laguna Mecoacán, Cowan 3199 (CICY, ENCB, TEX, XAL); Laguna de Mecoacán, López Portillo s.n. (IEB, ENCB, MEXU); Magaña A. 2443 (MEXU). Tamaulipas: Mpio. Aldama, zona inundable, Barra del Tordo, $1.2 \mathrm{~km}$ al O de la Playa, Mora O. 5471 (UAT); Mpio. Ciudad Madero, Playa Miramar, Torres G. s.n. (ANSM, ENCB, US); Mpio. Guerrero, $1 \mathrm{~km}$ al O del Rancho Las Anacahitas, González Medrano 628 (MEXU); Mpio. Matamoros, Wooton s.n. (US); $2 \mathrm{~km}$ al W de playa Bagdad, carretera playa BagdadMatamoros, Siqueiros 4908, 4909, 4910 (HUAA); 9 km sobre la brecha que va a la salinera, $4 \mathrm{~km}$ al $\mathrm{E}$ de Cinco de Mayo, sobre la brecha, $45 \mathrm{~km}$ al SE de Matamoros, Siqueiros 4911, 4912 (HUAA); $10 \mathrm{~km}$ de veinticinco de enero, Aguirre N. 7 (INEGI); $11 \mathrm{~km}$ al W de Playa Bagdad, sobre la carretera a Matamoros-Playa Bagdad, Siqueiros 4906, 4907 (HUAA); $14 \mathrm{~km}$ de la entrada a la brecha de Cinco de Mayo y $5 \mathrm{~km}$ al E de la Salinera, Siqueiros 4913 (HUAA); Charco temporal, $8 \mathrm{~km}$ al $\mathrm{O}$ de Playa Bagdad, Mora $O$. 10913 (UAT); Costa SE de Matamoros, LeSueur 670 (TEX); $\mathrm{km} 26$ al E de la carretera a la playa L. Villar, Baro D. et al. 241 (CICY, UAMIZ); Playa Washington, Marroquín 423 (UNL); Playa Washington a $38 \mathrm{~km}$ al E de Matamoros y 9 $\mathrm{km}$ al E sobre la playa, González Medrano 528 (MEXU); Rumbo a Playa Lauro Villar, Bores 43 (MEXU); zona inundable, $14 \mathrm{~km}$ de Matamoros rumbo a El Mezquital, Mora $O$. s.n. (UAT); Mpio. San Fernando, $2 \mathrm{~km}$ al W de General J. Mujica, $12.5 \mathrm{~km}$ sobre la brecha Santa Teresa J. Mujica, La Media Luna, Siqueiros 4914, 491, 4916, 4917 (HUAA); Alrededor de Media Luna, al SE del pueblo de pescadores de Santa Teresa por el faro cerca de la costa, Fryxell 3733 (ENCB, MEXU, TEX, US); Los Lirios, Galván 663 (ENCB); Punta de Alambre, Zuloaga et al. 9694 (ANSM); Mpio. Soto La Marina, $1.2 \mathrm{~km}$ antes de la Playa la Escollera, carr. Soto la Marina-La Presa, donde termina el camino, $\mathrm{Si}$ queiros 4921, 4922 (HUAA); $2 \mathrm{~km}$ al $\mathrm{N}$ de las Escolleras cerca de la Playa, ejido La Pesca, Mora O. 5437 (UAT); 4.8 $\mathrm{km}$ al E de Playa Grande, La Pesca, Nieto S. 358, 359, 360, 361, 362 (HUAA); $42 \mathrm{~km}$ sobre el camino Soto la MarinaLa Pesca, $7 \mathrm{~km}$ al E de Vista Hermosa, sobre el camino, en las márgenes de la Laguna Almagre, Siqueiros 4919 (HUAA); Barra de la Enramada $55 \mathrm{~km}$ al E de Ojo de Agua, Baro D. y col. 00768 (CICY); Brecha junto al Campamento Tortuguero, Playa Grande, La Pesca, Nieto S. 379, 380, 381, 382 (HUAA); Col. Miguel de la Madrid, Canal de la Bárcena, La Presa, Siqueiros 4920 (HUAA); Laguna Madre, Ejido la Pesca, Mora O. 7588 (UAT); La Pesca, Puig 2908 (ENCB); zona inundable, Estero Buena Vista, cerca de la Laguna Madre, Mora O. 7207 (UAT). Veracruz: Mpio. Alvarado, $0.5 \mathrm{~km}$ al S de Mandinga, Estero de Mandinga, Nieto $S .328,329,330,331,332$ (HUAA); $7.13 \mathrm{~km}$ al SE de Alvarado, islote en medio del Río Tlacotalpan, Encina et al. 3142 (ANSM); $11 \mathrm{~km}$ al N de Tlacotalpan, La Tunilla, Nieto S. 333, 334, 335, 336, 337 (HUAA); Laguna Alvarado, $\mathrm{Pa}$ - 
lestina et al. 1478 (XAL); Rincón La Palma, Campos y Pale 23396, 23489 (XAL); Río Acula, Castillo-Campos y Pale 23456, 23817 (XAL); Río Limón, Castillo-Campos y Pale 23455 (XAL); Mpio Pajapan, Jicacal, punta Chapotaj Isais s.n. (ANSM, ENCB, IEB); Mpio. Papaloapan, Matuda 28345 (MEXU, US, XAL); Mpio. Tecolutla, $2 \mathrm{~km}$ al $\mathrm{N}$ de Cruz de los Esteros (frente al puerto de Tecolutla), Nieto $S$. 323, 324, 325, 326, 327 (HUAA); Mpio. Tlacotalpan, cerca de la intersección de la carreteras 180 y 175, N de Tlacotalpan, Davidse y Davidse 9779 (MEXU); Paso de Obejas, Hernández X., X-3378 (US); Mpio. Tuxpan, Camino a la Hidroeléctrica de Tuxpan, orilla de la Laguna de Tampomachoco, $1.2 \mathrm{~km}$ a de la clínica del IMSS, $1.5 \mathrm{~km}$ del Puente Gabra el camino a la termoeléctrica, Siqueiros, 4924, 4925, 4926, 4927, 4928 (HUAA); Orilla del Río Tuxpan (suburbio Sur) Ave. Lázaro Cárdenas, lote baldío cerca de la desembocadura del río, Colonia Barra Norte, Siqueiros 4923 (HUAA); Vega del Río Tuxpan, Malecón Norte en la ciudad de Tuxpan, Nieto S. 318, 319, 320, 321, 322 (HUAA). Yucatán: Gaumer 24356 (US); Mpio. Hunucmá, $5 \mathrm{~km}$ al NO de Sisal, Nieto $S$. 348, 349, 350, 351, 352 (HUAA); Mpio. Progreso, $3 \mathrm{~km}$ al S de Progreso, Davidse y Davidse 29450 (MEXU); $4 \mathrm{~km}$ al S de Puerto Progreso, autopista Mérida- Pto. Progreso, Carr. No. 261, Nieto S. 214, 215, 216, 217, 218 (HUAA); 5 km de Progreso, Medina M. 2 (MEXU); 7.7 km S Chuburna Puerto. Carr. Sierra Papacal, Sabido Z. s.n. (INEGI); $17 \mathrm{~km}$ al E de Progreso, Gould 12616 (ENCB); Carretera Mérida-Progreso, circuito Chicxulub-Chelem, Ortiz 347 (CICY, CHAP, ENCB, XAL); Swallen 2921 (US); Mpio. Río Lagartos, 8 km al E de Rio Lagartos, Sabido Z. VPBA8 (INEG, UAMIZI); Mpio. San Felipe, $18 \mathrm{~km}$ al W de San Felipe, punta Bachul, Sabido Z. 24085 (INEGI); Mpio. Telchac Puerto, $1 \mathrm{~km}$ al S de Telchac Puerto (por la carr. No. 172), Nieto S. 219, 220, 221, 222 (HUAA); Telchac Puerto, Calzada et al. 6604 (CICY, MEXU, XAL); Mpio. Ucú, $6.6 \mathrm{~km}$ al S de Chuburna Puerto, Caballero M. 23 (INEGI); $7 \mathrm{~km}$ al S de Chuburna Puerto, Caballero M. 33 (INEGI).

\section{Especies dudosas y excluidas.}

Spartina cynosuroides (L.) Roth. Cat. Bot. 3:10. 1806. $=$ Dactylis cynosuroides $\mathrm{L} ., \equiv$ Trachynotia cynosuroides $(\mathrm{L}$.) Michx., $\equiv$ Paspalum cynosuroides (L.) Brot., झLimnetis cynosuroides (L.) Rich., $\equiv$ Cynodon cynosuroides (L.) Raspail. TIPO: Estados Unidos de Norte América, Virginia, Clayton 577 (Isolectotipo: BM; Sintipo: US).

Distribución y ecología.- Spartina cynosuroides crece en estuarios arenosos, en zonas de mareas de lagunas, bahías y pantanos. Se distribuye desde el este de las Costas del Golfo de México en Estado Unidos de Norte América. Para México fue citada por Espejo-Serna et al. (2000) en Tamaulipas y por Dávila-Aranda et al. (2006) para Chihuahua. Sin embargo, existe incertidumbre al respecto, dado que en las publicaciones donde se reporta no se proporcionan localida- des precisas ni respaldos de herbario y especialistas de los herbarios de la Ciudad de Chihuahua (Melgoza, com. pers.), desconocen la presencia de esta especie en el estado. Así mismo, no se encontraron ejemplares botánicos en ningún de los herbarios revisados, ni se localizaron en la visita de campo al estado de Chihuahua. El ejemplar R. Hernández N. 1881 (MEXU) de Tampico, identificado originalmente como $S$. cynosuroides, fue corregido a Spartina sp. por Angélica Rodríguez, ya que su hábito no corresponde a $S$. $c y$ nosuroides, además carece de estructuras reproductivas que permitan corroborar su identificación. Todos los ejemplares de esta especie que se revisaron fueron colectados en Estados Unidos de Norte América, por lo que la distribución en México de esta especie es dudosa.

Spartina gracilis Trin., Mém. Acad. Imp. Sci. Saint-Pétersbourg, Sér. 6, Sci. Math., Seconde Pt. Sci. Nat. 6,4(1-2): 110. 1845. TIPO: Canada. Amer. bor., Hooker s.n. (Isotipo: LE).

Distribución y ecología.- Se distribuye desde el noroeste y sur de Canadá hasta el centro de México (Barkworth 2003), en praderas alcalinas y en pantanos salinos y acequias. Espejo-Serna et al., (2000) reportan a Spartina gracilis para Jalisco y Michoacán y Dávila-Aranda et al. (2006), la mencionan para Chihuahua, no obstante, en ninguna de estas referencias bibliográficas se citan ejemplares de herbario ni localidades precisas. Además, en la revisión de ejemplares de herbario y en las exploraciones de campo no se logró encontrar a esta especie. Todos los especímenes examinados fueron de Texas, Estados Unidos de Norte América, por lo que no existen datos que confirmen su presencia en México.

A pesar de la exploración de campo y de herbarios llevada a cabo para este proyecto, no fue posible encontrar estas dos especies reportadas anteriormente para el país, por lo que se considera muy probable que dichos reportes hayan sido errores en la identificación del material en los herbarios. Sin embargo, no se descarta la posibilidad de que se encuentren algunas poblaciones en sitios no visitados, dada la vasta extensión de litorales en nuestro país.

Otras especies reportadas.

Existe un reporte de Spartina densiflora para Sinaloa (Faustino Hernández, A. Juan Gutiérrez y Rito Vega 231. 241 [UAS]), sin embargo, al revisar el ejemplar correspondiente y la población en el campo, en realidad es $S$. spartinae. Además, duplicados de estos ejemplares que están de intercambio en Herbario MEXU fueron determinados por Angélica Ramírez como $S$. spartinae.

\section{Agradecimientos}

Se agradece el apoyo de los curadores y personal de los herbarios por las facilidades brindadas para la consulta de sus ejemplares; a Rosa Cerros Tlatilpa, Yolanda Herrera Arrieta 
y Alicia Melgoza Castillo por la información proporcionada sobre distribución de Spartina; a Leopoldo Quezada Viay y a Alberto Rodríguez Ávalos por su apoyo en el trabajo de campo; a los revisores que con sus sugerencias se mejoró el manuscrito; al INEGI y la UAA por su apoyo para el desarrollo de este trabajo y al CONACYT por la beca 54764 otorgada a la primera autora.

\section{Literatura citada}

Ainouche M.L., Baumel A., Salmon A. y Yannic G. 2003. Hybridization, polyploidy and speciation in Spartina (Poaceae). New Phytologist 161:165-172.

Ainouche M.L., Fortune P.M., Salmon A., Parisod C., Grandbastien M.A., Fukunaga K., Ricou M. y Misset M.T. 2009. Hybridization, polyploidy and invasion: Lessons from Spartina (Poaceae). Biological Invasion 11:1159-1173.

Ayres D.R., Zaremba K., Sloop C.M. y Strong D. 2008. Sexual reproduction of cordgrass hybrids (Spartina foliosa $\times$ alterniflora) invading tidal marshes in San Francisco Bay. Diversity and Distributions 14:187-195.

Barkworth M.E. 2003. Spartina Schreb. En: Flora of North America Editorial Committe, Eds. Flora of North America, Vol. 25, pp. 240-251. Oxford University Press, Nueva York.

Baumel A., Ainouche M.L., Bayer R.J., Ainouche A.K. y Misset M.T. 2002. Molecular Phylogeny of Hybridizing Species from the Genus Spartina Schreb (Poaceae). Molecular Phylogenetics and Evolution 22:303-314.

Baumel A., Ainouche M.L., Misset M.T., Gourret J-P. y Bayer R.J. 2003. Genetic evidence for hybridization between the native Spartina maritima and the introduced Spartina alterniflora (Poaceae) in South-West France: Spartina X neyrautii re-examined. Plant Systematics and Evolution 237:89-97.

Correll D.S. y Correll H.B. 1972. Aquatic and Wetland Plants of Southwestern United States. Enviromental Protection Agency. Washington DC.

Correll D.S. y Johnston M.C. 1970. Manual of the Vascular Plants of Texas. Texas Research Foudation. Texas.

Dávila-Aranda P., Mejía-Saulés M.T., Gómez-Sánchez M., ValdésReyna J., Ortiz J.J., Morín C., Castrejón J. y Ocampo A. 2006. Catálogo de gramíneas de México. Universidad Nacional Autónoma de México y Comisión Nacional para el Conocimiento y Uso de la Biodiversidad, México D.F.

Espejo-Serna S.A., López-Ferrari A.R. y Valdés-Reyna J. 2000. Poaceae. En: Espejo-Serna A. y López-Ferrari A.R. Eds. Las Monocotiledoneas de México: Sinopsis Florística, Partes IXXI 10, pp. 211-212. Consejo Nacional de la Flora de México A.C., Universidad Autónoma Metropolitana-Iztapalapa, and Comisión Nacional para el Conocimiento y uso de la Biodiversidad, México D.F.

Espinar J.L. 2009. Pastizales de Spartina (Spartinion maritimae). En: W.AA. Bases ecológicas preliminares para la conservación de los tipos de hábitat de interés comunitario en España, pp. 7-12. Ministerio de Medio Ambiente, y Medio Rural y Marino, Madrid. <http://www.magrama.gob.es/es/biodiversidad/temas/ espacios-protegidos/1320_tcm7-24037.pdf > (consultado 10 diciembre 2012)

Fortune P.M., Schierenbeck K., Ayres D., Bortolus A., Catrice O., Brown S. y Ainouche M.L. 2008. The enigmatic invasive Spar- tina densiflora: A history of hybridizations in a polyploidy context. Molecular Ecology 17:4304-4316.

Gedan K.B. y Bertness M.D. 2010. How will warming affect the salt marsh foundation species Spartina patens and its ecological role? Oecologia 164:479-487.

Gould F.W. 1975. The Grasses of Texas. Texas A \& M University Press, College Station.

Gould F.W. y Moran R. 1981. The grasses of Baja California, Mexico. Memoirs of the San Diego Society of Natural History Vol. 12, San Diego.

Herrera-ArrietaY. y Cortés-Ortíz A. 2010. Listado florístico y aspectos ecológicos de la familia Poaceae para Chihuahua, Durango y Zacatecas, México. Journal of the Botanical Research Institute of Texas 4:711-738.

Hitchcock A.S. 1971. Manual of the Grasses of the United States. (Vol I.) Dover Publications Inc., Nueva York.

Hogle I. 2011. San Francisco Estuary Invasive Spartina Project: Comparison of 2010 Field and Lab Identification of Spartina at Cooley Landing Restoration Project, East Palo Alto, CA. State Coastal Conservancy, Oakland. <http://www.spartina.org/documents/ISPMonApproachReport_fin_w\%20Appendix.pdf> (consultado 20 febrero 2012)

INEGI. 2013. Conjunto de datos vectoriales de Uso del Suelo y Vegetación escala 1:250 000 serie V. Instituto Nacional de Estadística y Geografía, México. <http://www.inegi.org.mx/geo/ contenidos/recnat/usosuelo/> (consultado 5 mayo 2013)

Kearney T.H. y Peebles R.H. 1960. Arizona Flora. University of California Press.

Kim S., Rayburn A.L., Parrish A. y Lee D.K. 2012. Cytogeographic distribution and genome size variation in prairie cordgrass (Spartina pectinata Bosc ex Link). Plant Molecular Biology Reporter 30:1073-1079.

Lot A. y Chiang, F. 1986. Manual del Herbario. Administración y manejo de colecciones, técnicas de recolección y preparación de ejemplares botánicos. Consejo Nacional de la Flora de México, A. C. México D.F.

Mora-Olivo A. y Valdés-Reyna J. 2011. Nota sobre la presencia de Spartina alterniflora Loisel. (Poaceae: Chloridoideae: Cynodonteae) en humedales mexicanos. Acta Botanica Mexicana 95:45-50.

Mobberley, D.G. 1956. Taxonomy and distribution of the genus Spartina. Iowa State College. Journal of Science 30:471-574.

Peterson P.M., Soreng R.J., Davidse G., Filgueiras T.S., Zuloaga F.O. y Judziewicz E.J. 2001. Catalogue of New World grasses (Poaceae) II. Subfamily Chloridoideae. Contributions from the United States National Herbarium Smithsonian Institution. 41:1-255.

Peterson P.M., Romaschenko K. y Johnson G. 2010. A classification of the Chloridoideae (Poaceae) based on multi-gene phylogenetics trees. Molecular Phylogenetics and Evolution 55:580-598.

Peterson P.M., Romaschenko K., Herrera-Arrieta Y. y Saarela J.M. 2014. A molecular phylogeny and new subgeneric classification of Sporobolus (Poaceae: Chloridoideae: Sporobolinae). Taxon 63: $1212-1243$.

Pohl R.W. 1994. Spartina. En: Davidse G., Sousa-Sánchez M. y Chater A.O. Eds. Flora Mesoamericana. Vol. 6, pp. 292. Instituto de Biología. Universidad Nacional Autónoma de MéxicoMissouri Botanical Gardner. México D.F.

Saarela J.M. 2012. Taxonomic synopsis of invasive and native 
Spartina (Poaceae, Chloridoideae) in the Pacific Northwest (British Columbia, Washington and Oregon), including the first report of Spartina $\times$ townsendii for British Columbia, Canada. Phytokeys 10:25-82.

Shreve F. y Wiggins I.L. 1964. Vegetation and flora of the Sonora Desert. Stanford University Press, Stanford. United States of America.

Soreng R.J., Davidse G., Peterson P.M., Zuloaga F.O., Judziewicz E.J., Filgueiras T.S. y Morrone O. 2003. On-line taxonomic novelties and updates, distributional additions and corrections, and editorial changes since the four published volumes of the Catalogue of New World Grasses (Poaceae) published in Contr. U.S. Natl. Herb. vols. 39, 41, 46, and 48. En: Soreng R.J., Davidse G., Peterson P.M., Zuloaga F.O., Filgueiras T.S.,
Judziewicz E.J. y Morrone O. Eds. Internet Cat. New World Grasses. Missouri Botanical Garden, St. Louis. <http://www. tropicos.org/Project/CNWG > (consultado 6 abril 2012)

Sosa V. y Gómez-Pompa A. 1994. Lista florística. Flora de Veracruz. Instituto de Ecología, A.C, Xalapa.

Swallen, J.R y McClure F.A. 1955. Flora of Guatemala, part. II: Grasses of Guatemala: Bamboos Fieldiana. Botany, Vol. 24 (part 2). Chicago Natural History Museum Press, Chicago

Wiggins I.L. 1980. Flora of Baja California. Standford University Press, Stanford.

Zhi Y., Li H., An S., Zhao L., Zhou C. y Deng Z. 2007. Interspecific competition: Spartina alterniflora is replacing Spartina anglica in coastal China. Estuarine, Coastal and Shelf Science 74:437-448.

Recibido: 19 de marzo de 2014

Aceptado: 6 de agosto de 2014 
El GÉNero Spartina EN MÉxico

Apéndice 1. Localidades de México muestreadas para este proyecto ( $\mathrm{msnm}=$ metros sobre el nivel del mar).

\begin{tabular}{|c|c|c|c|c|c|c|}
\hline Localidad & Municipio & Estado & Hábitat & Longitud & Latitud & msnm \\
\hline $\begin{array}{l}\text { Boca de Estero Beach, en los limites } \\
\text { sur de la Ciudad de Ensenada (enfrente } \\
\text { del Hotel-Resorts Estero Beach y la } \\
\text { playa de Estero Beach) }\end{array}$ & Ensenada & Baja California & Manglar & $116^{\circ} 36^{\prime} 41.57^{\prime \prime W}$ & $31^{\circ} 46^{\prime} 32.17^{\prime \prime} \mathrm{N}$ & 0 \\
\hline $\begin{array}{l}\text { Puerto Adolfo López Mateos, estero } \\
\text { en el margen del manglar }\end{array}$ & Comondú & $\begin{array}{l}\text { Baja California } \\
\text { Sur }\end{array}$ & Manglar & $112^{\circ} 04^{\prime} 41.60^{\prime \prime} \mathrm{W}$ & $25^{\circ} 15^{\prime} 06.00^{\prime \prime} \mathrm{N}$ & 1 \\
\hline $\begin{array}{l}\text { Puerto San Carlos, entrada E del } \\
\text { Pueblo, carr. Constitución-San Carlos, } \\
\text { playa a la entrada }\end{array}$ & Comondú & $\begin{array}{l}\text { Baja California } \\
\text { Sur }\end{array}$ & $\begin{array}{l}\text { Vegetación } \\
\text { acuática }\end{array}$ & $112^{\circ} 06^{\prime} 54.20^{\prime \prime} \mathrm{W}$ & $24^{\circ} 47^{\prime} 57.20^{\prime \prime} \mathrm{N}$ & 0 \\
\hline $\begin{array}{l}\text { Puerto Cancún } 0.5 \text { km al W del Pueblo, } \\
32 \mathrm{Km} \text { del entronque las Brisas Carr. } \\
\text { La Paz-Cd. Constitución }\end{array}$ & La Paz & $\begin{array}{l}\text { Baja California } \\
\text { Sur }\end{array}$ & $\begin{array}{l}\text { Vegetación } \\
\text { acuática }\end{array}$ & $111^{\circ} 44^{\prime} 23.80^{\prime \prime} \mathrm{W}$ & $24^{\circ} 32^{\prime} 55.30^{\prime \prime} \mathrm{N}$ & 0 \\
\hline $\begin{array}{l}\text { Puerto Chale, Estero de Puerto Chale, } \\
\text { frente a la orilla del Estero. }\end{array}$ & La Paz & $\begin{array}{l}\text { Baja California } \\
\text { Sur }\end{array}$ & $\begin{array}{l}\text { Vegetación } \\
\text { acuática }\end{array}$ & $111^{\circ} 33^{\prime} 16.90^{\prime \prime} \mathrm{W}$ & $24^{\circ} 25^{\prime} 18.10^{\prime \prime} \mathrm{N}$ & 6 \\
\hline $\begin{array}{l}6.8 \mathrm{~km} \text { al NW de Punta Abreojos, } \\
\text { estero La Bocana, extremo sur } \\
\text { márgenes del estero }\end{array}$ & Mulegé & $\begin{array}{l}\text { Baja California } \\
\text { Sur }\end{array}$ & $\begin{array}{l}\text { Dunas } \\
\text { costeras }\end{array}$ & $113^{\circ} 38^{\prime} 18.70^{\prime \prime} \mathrm{W}$ & $26^{\circ} 45^{\prime} 02.50^{\prime \prime} \mathrm{N}$ & 6 \\
\hline $\begin{array}{l}\text { Canal que cruza la Cd de Guerrero } \\
\text { Negro perpendicular a la calle principal } \\
\text { sobre los margenes del canal }\end{array}$ & Mulegé & $\begin{array}{l}\text { Baja California } \\
\text { Sur }\end{array}$ & $\begin{array}{l}\text { Vegetación } \\
\text { acuática }\end{array}$ & $114^{\circ} 03^{\prime} 14.00^{\prime \prime} \mathrm{W}$ & $27^{\circ} 57^{\prime} 50.10^{\prime \prime} \mathrm{N}$ & 24 \\
\hline $\begin{array}{l}\text { Laguna Ojo de Liebre, } 5 \mathrm{~km} \text { al NW } \\
\text { del pueblo de Guerrero Negro, Brecha } \\
\text { al Faro Viejo }\end{array}$ & Mulegé & $\begin{array}{l}\text { Baja California } \\
\text { Sur }\end{array}$ & $\begin{array}{l}\text { Pastizal } \\
\text { halófilo }\end{array}$ & $114^{\circ} 04^{\prime} 50.40^{\prime \prime} \mathrm{W}$ & $27^{\circ} 58^{\prime} 28.70^{\prime \prime} \mathrm{N}$ & 6 \\
\hline $\begin{array}{l}11 \mathrm{~km} \text { al SE de la Reserva de la Biosfera } \\
\text { Los Petenes (terracería a la Isla Jaina) }\end{array}$ & Calkiní & Campeche & Manglar & $090^{\circ} 25^{\prime} 54.40^{\prime \prime} \mathrm{W}$ & $20^{\circ} 10^{\prime} 56.40^{\prime \prime} \mathrm{N}$ & 3 \\
\hline $\begin{array}{l}0.5 \mathrm{~km} \text { al NE de Campeche (por la } \\
\text { salida a Carr. Campeche-Mérida) }\end{array}$ & Campeche & Campeche & Manglar & $090^{\circ} 29^{\prime} 15.30^{\prime \prime} \mathrm{W}$ & $19^{\circ} 52^{\prime} 32.10^{\prime \prime} \mathrm{N}$ & 2 \\
\hline $\begin{array}{l}10 \mathrm{~km} \text { al E de Cd. Del Carmen (frente } \\
\text { a la finca Mayaraki) km } 19 \text { de la carr. } \\
\text { Cd. del Carmen-Campeche }\end{array}$ & Carmen & Campeche & Manglar & $091^{\circ} 41^{\prime} 17.50^{\prime \prime} \mathrm{W}$ & $18^{\circ} 42^{\prime} 11.70^{\prime \prime} \mathrm{N}$ & 15 \\
\hline $\begin{array}{l}\text { Ejido Puerto Rico, } 7 \text { km al N del Zacatal, } \\
\text { km } 16 \text { carr. Villahermosa-Cd. del Carmen, }\end{array}$ & Carmen & Campeche & Manglar & $091^{\circ} 53^{\prime} 05.80^{\prime \prime} \mathrm{W}$ & $18^{\circ} 37^{\prime} 12.00^{\prime \prime} \mathrm{N}$ & 0 \\
\hline $\begin{array}{l}0.5 \mathrm{~km} \text { al S de Sabancuy (orillas del } \\
\text { pueblo de Sabancuy por la salida } \\
\text { a Chicbul) }\end{array}$ & Champotón & Campeche & $\begin{array}{l}\text { Pastizal } \\
\text { halófilo }\end{array}$ & $091^{\circ} 10^{\prime} 30.50^{\prime \prime} \mathrm{W}$ & $18^{\circ} 57^{\prime} 59.70^{\prime \prime} \mathrm{N}$ & 0 \\
\hline $\begin{array}{l}\text { Km } 53 \text { de la autopista } \mathrm{Cd} \text {. del } \\
\text { Carmen-Champoton }\end{array}$ & Champotón & Campeche & Manglar & $091^{\circ} 23^{\prime} 27.00^{\prime \prime} \mathrm{W}$ & $18^{\circ} 53^{\prime} 30.10^{\prime \prime} \mathrm{N}$ & 2 \\
\hline $\begin{array}{l}1.2 \mathrm{~km} \text { al SE de Paredón, a la orilla } \\
\text { del pueblo }\end{array}$ & Tonalá & Chiapas & Manglar & $093^{\circ} 52^{\prime} 03.48^{\prime \prime} \mathrm{W}$ & $16^{\circ} 02^{\prime} 35.20^{\prime \prime} \mathrm{N}$ & 1 \\
\hline $2.5 \mathrm{~km}$ al $\mathrm{W}$ de Puerto Arista & Tonalá & Chiapas & $\begin{array}{c}\text { Dunas } \\
\text { costeras }\end{array}$ & $093^{\circ} 50^{\prime} 05.18^{\prime \prime} \mathrm{W}$ & $15^{\circ} 56^{\prime} 45.75^{\prime \prime} \mathrm{N}$ & 0 \\
\hline
\end{tabular}


Apéndice 1. Continuación.

\begin{tabular}{|c|c|c|c|c|c|c|}
\hline Localidad & Municipio & Estado & Hábitat & Longitud & Latitud & msnm \\
\hline $\begin{array}{l}\text { Salinera de Estación Carrillo, a } 1.5 \text { km } \\
\text { al E de Carrillo, alrededores de la } \\
\text { Laguna Palomas }\end{array}$ & Jiménez & Chihuahua & $\begin{array}{l}\text { Pastizal } \\
\text { halófilo }\end{array}$ & $103^{\circ} 55^{\prime} 04.00^{\prime \prime} \mathrm{W}$ & $26^{\circ} 53^{\prime} 22.26^{\prime \prime} \mathrm{N}$ & 1099 \\
\hline $\begin{array}{l}\text { Rancho el Fresnal, al SW del cerro a } \\
10 \mathrm{~km} \text { al } \mathrm{N} \text { de el Sabinal, alrededores } \\
\text { de Laguna Janos }\end{array}$ & Asención & Chihuahua & $\begin{array}{l}\text { Matorral } \\
\text { micrófilo }\end{array}$ & $107^{\circ} 31^{\prime} 34.60^{\prime \prime} \mathrm{W}$ & $31^{\circ} 02^{\prime} 41.35^{\prime \prime} \mathrm{N}$ & 1187 \\
\hline $\begin{array}{l}5 \mathrm{~km} \text { al E de Nuevos Casas Grandes, } \\
\text { alrededores de la Laguna Fierros. }\end{array}$ & $\begin{array}{l}\text { Nuevo Casas } \\
\text { Grandes }\end{array}$ & Chihuahua & $\begin{array}{l}\text { Matorral } \\
\text { espinoso }\end{array}$ & $107^{\circ} 51^{\prime} 18.12^{\prime \prime} \mathrm{W}$ & $30^{\circ} 23^{\prime} 51.21^{\prime \prime} \mathrm{N}$ & 1450 \\
\hline Poza azul & Cuatrociénegas & Coahuila & $\begin{array}{l}\text { Pastizal } \\
\text { halófilo } \\
\text { (espartinal) }\end{array}$ & $102^{\circ} 07^{\prime} 20.30^{\prime \prime} \mathrm{W}$ & $26^{\circ} 55^{\prime} 17.80^{\prime \prime} \mathrm{N}$ & 726 \\
\hline $\begin{array}{l}2 \mathrm{~km} \text { al } \mathrm{N} \text { de las tablas } 9.5 \mathrm{~km} \text { al } \mathrm{S} \text { de } \\
\text { Moritas, al } \mathrm{SW} \text { de Cd. del Maíz }\end{array}$ & Cd. del Maíz & $\begin{array}{l}\text { San Luis } \\
\text { Potosí }\end{array}$ & $\begin{array}{l}\text { Pastizal } \\
\text { halófilo } \\
\text { (espartinal) }\end{array}$ & $099^{\circ} 51^{\prime} 51.01^{\prime \prime} \mathrm{W}$ & $22^{\circ} 16^{\prime} 26.64^{\prime \prime} \mathrm{N}$ & 1031 \\
\hline $\begin{array}{l}\text { El Conchal, } 0.2 \mathrm{~km} \text { al } \mathrm{W} \text { de la carr. al } \\
\text { Conchal, a la entrada } \mathrm{S} \text { del pueblo }\end{array}$ & Culiacán & Sinaloa & Manglar & $107^{\circ} 20^{\prime} 22.50^{\prime \prime} \mathrm{W}$ & $24^{\circ} 15^{\prime} 38.10^{\prime \prime} \mathrm{N}$ & 13 \\
\hline $\begin{array}{l}15 \mathrm{~km} \text { al } \mathrm{W} \text { del Dorado (sobre brecha } \\
\text { a los Algodones, playa de Ponce) }\end{array}$ & Culiacán & Sinaloa & $\begin{array}{l}\text { Zona } \\
\text { inundable en } \\
\text { laro de mangla }\end{array}$ & $107^{\circ} 35^{\prime} 08.00^{\prime \prime} \mathrm{W}$ & $24^{\circ} 22^{\prime} 26.90^{\prime \prime} \mathrm{N}$ & 2 \\
\hline $\begin{array}{l}7 \mathrm{~km} \text { al } \mathrm{N} \text { de Frontera (Carr. a } \\
\text { Frontera - Playas el Bosque }\end{array}$ & Centla & Tabasco & Manglar & $092^{\circ} 39^{\prime} 34.30^{\prime \prime} \mathrm{W}$ & $18^{\circ} 35^{\prime} 58.70^{\prime \prime} \mathrm{N}$ & 0 \\
\hline $\begin{array}{l}1.5 \mathrm{~km} \text { al } \mathrm{S} \text { de Chiltepec en la } \\
\text { Laguna Mecoacán (por la carr. } \\
\text { Chiltepec-Nicolás Bravo). }\end{array}$ & Paraíso & Tabasco & Manglar & $093^{\circ} 04^{\prime} 39.10^{\prime \prime} \mathrm{W}$ & $18^{\circ} 24^{\prime} 43.50^{\prime \prime} \mathrm{N}$ & 8 \\
\hline $\begin{array}{l}\text { Rio Soto la Marina, sobre el margen } \\
\text { del río en el hotel Blanquita }\end{array}$ & Soto la Marina & Tamaulipas & $\begin{array}{l}\text { Vegetación } \\
\text { acuática }\end{array}$ & $097^{\circ} 46^{\prime} 11.40^{\prime \prime} \mathrm{W}$ & $23^{\circ} 47^{\prime} 11.20^{\prime \prime} \mathrm{N}$ & 2 \\
\hline $\begin{array}{l}\text { Brecha junto al campamento } \\
\text { tortuguero, Playa Grande, } 1 \mathrm{~km} \\
\text { al N del espartinal }\end{array}$ & Soto La Marina & Tamaulipas & $\begin{array}{l}\text { Dunas } \\
\text { costeras }\end{array}$ & $097^{\circ} 44^{\prime} 12.50^{\prime \prime} \mathrm{W}$ & $23^{\circ} 47^{\prime} 27.80^{\prime \prime} \mathrm{N}$ & 0 \\
\hline $\begin{array}{l}\text { La Playita, margen del estero } \\
\text { frente a la playita }\end{array}$ & Soto La Marina & Tamaulipas & $\begin{array}{l}\text { Dunas } \\
\text { costeras }\end{array}$ & $097^{\circ} 44^{\prime} 13.40^{\prime \prime} \mathrm{W}$ & $23^{\circ} 46^{\prime} 24.00^{\prime \prime} \mathrm{N}$ & 0 \\
\hline $\begin{array}{l}11 \mathrm{~km} \text { al W de Playa Bagdad, sobre la } \\
\text { carretera a Matamoros-Playa Bagdad } \\
\text { zona inundable a orilla de carretera }\end{array}$ & Matamoros & Tamaulipas & $\begin{array}{c}\text { Zona } 0 \\
\text { undable a oril } \\
\text { de carretera }\end{array}$ & $\begin{array}{l}097^{\circ} 15^{\prime} 009.80^{\prime \prime} \mathrm{W} \\
\text { Illa }\end{array}$ & $25^{\circ} 49^{\prime} 12.70^{\prime \prime} \mathrm{N}$ & 1 \\
\hline $\begin{array}{l}14 \mathrm{~km} \text { de la entrada a la brecha de } \\
\text { Cinco de Mayo y } 5 \mathrm{~km} \text { al E de la Salinera }\end{array}$ & Matamoros & Tamaulipas & $\begin{array}{l}\text { Pastizal } \\
\text { halófilo }\end{array}$ & $097^{\circ} 33^{\prime} 13.31^{\prime \prime} \mathrm{W}$ & $25^{\circ} 30^{\prime} 44.52^{\prime \prime} \mathrm{N}$ & 1 \\
\hline $\begin{array}{l}2 \mathrm{~km} \text { al W de Playa Bagdad, carr. Playa } \\
\text { Bagdad-Matamoros, orilla de carretera }\end{array}$ & Matamoros & Tamaulipas & $\begin{array}{l}\text { Zona } \\
\text { inundable con } \\
\text { Prosopis }\end{array}$ & $097^{\circ} 10^{\prime} 36.02^{\prime \prime} \mathrm{W}$ & $25^{\circ} 48^{\prime} 57.33^{\prime \prime} \mathrm{N}$ & 1 \\
\hline $\begin{array}{l}9 \mathrm{~km} \text { sobre la brecha que va a la } \\
\text { salinera, } 4 \mathrm{~km} \text { al E de Cinco de Mayo } \\
\text { sobre la brecha, } 45 \mathrm{~km} \text { al SE } \\
\text { de Matamoros }\end{array}$ & Matamoros & Tamaulipas & $\begin{array}{l}\text { Zona } \\
\text { inundable en } \\
\text { atorral espinos }\end{array}$ & $097^{\circ} 35^{\prime} 10.11^{\prime \prime} \mathrm{W}$ & $25^{\circ} 31^{\prime} 54.14^{\prime \prime} \mathrm{N}$ & 1 \\
\hline
\end{tabular}


El GÉNERo SPARTINA EN MÉXICO

Apéndice 1. Continuación.

\begin{tabular}{|c|c|c|c|c|c|c|}
\hline Localidad & Municipio & Estado & Hábitat & Longitud & Latitud & msnm \\
\hline $\begin{array}{l}2 \mathrm{~km} \text { al W de General J. Mujica, } \\
12.5 \mathrm{~km} \text { sobre la brecha Sta. Teresa-J. } \\
\text { Mujica, La Media Luna }\end{array}$ & San Fernando & Tamaulipas & $\begin{array}{c}\text { Zona } \\
\text { inundable } \\
\text { con Prosopis }\end{array}$ & $097^{\circ} 45^{\prime} 44.83^{\prime \prime} \mathrm{W}$ & $25^{\circ} 14^{\prime} 44.71^{\prime \prime} \mathrm{N}$ & 9 \\
\hline $\begin{array}{l}1.2 \mathrm{~km} \text { antes de la Playa la Escollera, } \\
\text { carr. Soto la Marina-La Pesca donde } \\
\text { termina el camino a los lados de }\end{array}$ & Soto La Marina & Tamaulipas & $\begin{array}{l}\text { Pastizal } \\
\text { halófilo } \\
\text { (espartinal) }\end{array}$ & $097^{\circ} 44^{\prime} 25.50^{\prime \prime} \mathrm{W}$ & $23^{\circ} 46^{\prime} 54.14^{\prime \prime} \mathrm{N}$ & 4 \\
\hline
\end{tabular}

la carretera.

$4.8 \mathrm{~km}$ al E de Playa Grande,

La Pesca
Soto La Marina Tamaulipas zona $097^{\circ} 45^{\prime} 44.80^{\prime \prime} \mathrm{W} \quad 23^{\circ} 47^{\prime} 09.10^{\prime \prime} \mathrm{N} \quad 9$ arenosa, márgenes del estero
Soto La Marina Tamaulipas zona $097^{\circ} 48^{\prime} 309.04^{\prime \prime} \mathrm{W} \quad 23^{\circ} 47^{\prime} 51.72^{\prime \prime} \mathrm{N} \quad 1$ inundable en claro de manglar

$42 \mathrm{~km}$ sobre el camino Soto la Marina-La Pesca, 7 km al E de Vista Hermosa, sobre el camino, en las márgenes de la laguna Almagre

Col. Miguel de la Madrid, Canal de la Barcena, La Pesca

Vega del Río Tuxpan, Malecón Norte en la ciudad de Tuxpan

$0.5 \mathrm{~km}$ al S de Mandinga, Estero de Mandinga

11 km al N de Tlacotalpan, La Tunilla

$2 \mathrm{~km}$ al $\mathrm{N}$ de Cruz de los Esteros

(frente al puerto de Tecolutla)

Camino a la Hidroeléctrica de Tuxpan, orilla de la Laguna de Tampomachoco, $1.2 \mathrm{~km}$ de la clínica del IMSS, $1.5 \mathrm{~km}$ del Puente Gabra en el camino a la termoeléctrica

Orilla del Río Tuxpan (Suburbio Sur), Ave. Lázaro Cárdenas, lote baldío cerca de la desembocadura del río, Colonia Barra Norte

$5 \mathrm{~km}$ al NW de Sisal

$4 \mathrm{~km}$ al S de Puerto Progreso (autopista Mérida- Pto. Progreso, Carr. No. 261)

$1 \mathrm{~km}$ al S de Puerto Telchac (por la carr. No. 172)
Soto La Marina Tamaulipas

Tuxpan Veracruz

Alvarado Veracruz

Alvarado Veracruz

Tecolutla Veracruz

Tuxpan

Veracruz

Tuxpan

Veracruz

Hunucmá Yucatán

Progreso Yucatán

Telchac Puerto Yucatán
Márgenes

del canal

Vegetación $\quad 097^{\circ} 22^{\prime} 26.80^{\prime \prime} \mathrm{W} \quad 20^{\circ} 56^{\prime} 29.20^{\prime \prime} \mathrm{N} \quad-8$ acuática

Manglar

$096^{\circ} 20^{\prime} 05.80^{\prime \prime} \mathrm{W} \quad 1^{\circ} 24^{\prime} 54.00^{\prime \prime} \mathrm{N}$

0

Manglar

$095^{\circ} 38^{\prime} 34.28^{\prime \prime} \mathrm{W} \quad 18^{\circ} 42^{\prime} 28.82^{\prime \prime} \mathrm{N}$

Pastizal halófilo

(espartinal)

$097^{\circ} 00^{\prime} 59.90^{\prime \prime} \mathrm{W} \quad 20^{\circ} 27^{\prime} 45.30^{\prime \prime} \mathrm{N} \quad 1$

zona urbana

$097^{\circ} 09^{\prime} 04.37^{\prime \prime} \mathrm{W} \quad 20^{\circ} 58^{\prime} 55.44^{\prime \prime} \mathrm{N}$

1

Vegetación acuática

$097^{\circ} 21^{\prime} 38.72^{\prime \prime} \mathrm{W} \quad 20^{\circ} 56^{\prime} 43.31^{\prime \prime} \mathrm{N}$

1

Manglar

$090^{\circ} 01^{\prime} 07.10^{\prime \prime} \mathrm{W} \quad 21^{\circ} 08^{\prime} 59.90^{\prime \prime} \mathrm{N}$

13

Manglar

$089^{\circ} 39^{\prime} 32.00^{\prime \prime} \mathrm{W} \quad 21^{\circ} 14^{\prime} 39.00^{\prime \prime} \mathrm{N}$

7

Pastizal

halófilo

(espartinal) 